\title{
Zur Psychologie der Weltanschauungen ${ }^{\text {}) . ~}$ Von Jonass Cohn.
}

Das Werk von Jaspers bietet der nachprüfenden Betrachtang eine Mehrheit von Ansichten. Es enthält im Kerne ein Hanptstïck verstehender Psychologie, eine nachfiihlende Schildernng der Weltanschanungen als des Ausdracks and der Lebensformen bestimmter geistiger Typèn. Aber mit dieser mehr deskriptiven Aufgabe begnügt sich Jaspers nicht; er geht von den offen liegenden Weltanschanungen zarück auf die sich in ihnen auswirkenden Kräfte, die er mit bemerkenswerter Fortbildung kantischer Lehren "Ideen" nennt, und auf das unfaßbare "Leben", das alles trägt und erzengt. Als kritischer Denker erstrebt er dann Verständnis seines eigenen Tuns; daher enthält das Buch Beiträge zu der Analyse des Verstehens, das die Methode seines Forschens bildet, und der Dialektik, mit deren Hilfe seine Darstellung sich des "Lebens" za bemächtigen sucht. Dieses Bemühen um Erkenntnis and Einordnung seiner Arbeit beschränkt sich aber, wie billig, nicht auf den Weg; sondern wendet sich auch den Zielen zu. Er bemüht sich,' seine Anfgabe als wertfreie Betrachtung der Welt-

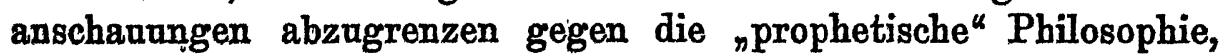
die. Weltanschauung geben will, and ihr im Ganzen der Psychologie und zum Ganzen der Philosophie ihre Stelle anzuweisen. Je nach der Seite, von der aus man das Werk ansieht, verschiebt sich die relative Wichtigkeit der Teile; man gerät in Gefahr, Wesentliches zu übersehen und die Bedeutung des Werkes ungenügend zu würdigen. Daher seien im Folgenden die vier genannten Ansichten nacheinander vorgeführt.

Die leitende Idee des Buches ist die eines geordneten Systems,

1) Jaspers, Karl, Psychologie der Weltanschauungen. Berlin, Julius 'Springer, 1919, XII u. 428 S: Die Besprechung wurde geschrieben, ehe Hein- a rich Rickert's Abhandlung: Psychologie der Weltanschaungen und Philosophie der Werte (Logos IX, 1, 1920) und dessen Buch: Philosophie des Lebens, Túbingen 1920 erschienen war. Es war daher leider nicht möglich, diese Arbeiten zu berücksichtigen. 
eines „Kosmos der Weltanschaungen " (22). Eine Aufgabè, die sich schon Dilthey stellte, wird in umfassenderer Art zu lösen gesacht. Unter "Weltansehauung" versteht dabei J. kein nur contemplatives Verhalten, sie ist vielmehr dạs Ganze aus Wissen, Wertangen, Impalsen, in dem sich „das Letzth und das Totale des Menschen sowobl subjektiv als Erlebnis and Krátt. and Gesinnung wie objektiv als gegenständlich gestaltete Welt" darstellt (1). Eine Weltanschanung kann nun dem Mẹnschen selbstverständlich gegeben sein, er kann schlicht in ihr leben, wie das in gebandenen Zeiten and Kalturen die Regel ist. Von diesem Auftreten der Weltanschanüngen, das nor sozialpsychologisch, nicht individualpsychologisch za begreifen ist, will J. micht reden. Erst wo die überlieferten Bindungen, Beschränkangen, Heimaten (alles was J. „Gehäuse " nennt) sich auflösên, tritt das Leben ein', das Gegenstand einer Weltanschauungspsychologie des Individuams sein kann (248). Dabei zeigt sich das Leben, die "Idee" der Weltanschanung, nar in wenigen Fällen ganz ursprünglich; stark, echt; um jede dieser „substantiellen" Weltanschanungen als Żentrum gruppieren sich verwandte aber verkümmerte Gestalten (27). Zu ihnen gehören die ${ }_{n}$ unechten ${ }^{\prime}$ Formen. Das Unechte ist ${ }_{n}$ nicht Uñwirklichkeit aber Wirkangslosigkeit, nicht Lïge aber gleichșam organische Verlogenheit" (32). Sehwindet die lebendige Einheit von Form und Gehalt, so entstehen „formalisierte" Gestalten, in der Kunst z. B. die Artistik. Den diffexenzierten Formen stehen vorbereitend oder begleitend undifferenzierte zar Seite. Endlich kann an Stelle der lebendigen Weltanschaung die bloBe Formel gesetzt werden, der Fanatiker behauptet starr sein Credo. J. geht überall von den echten, lebendigen, differenzierten Gestalten aus, sucht dann ron ihnen ans die Prozesse der Verkümmerang zn verstehen.

Als Leitfaden in der Mannigfaltigkeit der Weltanschanungen and als Einteilangsprinzip dient das Urphänomen der Sabjekt-Objekt-Spaltung (22). Subjekt, Objekt and die Beziehang zwischen ihnen sind aber nicht feste Gebilde, sondern nehmen sehr verschiedene Gestalten and Bedentangen an, die in einander übergehen oder sich gegen einander abgrenzen. Von der Subjektseite her gesehen sind die Weltanschaunngen "Einstellnngen", von der Objektseite her "Weltbilder". Aber das sind blob Elemente - in das Zentrum gelangt man erst mit der Betrachtung der "Geistestypen" „die amfassend Weltbilder and Einstellungen in sich schließen, die nicht anmittelbar za vergegenwärtigen sind, wie alle jene Elemente, 
sondern vielmehr nur als Bewegungsprozesse, als Totalitäten, denen eine treibende Kraft za Grande liegt" (39). Die Darstellung dieser Grundlagen, das allgemeine Schema, ist der Gegenstand dieses Buches. Eine vollständige Psychologie der Weltanschaungen würde anßerdem noch zwei Teile umfassen; der zweite Teil müßte diese Kategorien in die einzelnen Gebiete verfolgen, in die Werksphäre (z. B.: Wissenschaft, Metaphysik, Kanst, Religion), in die Persönlichkeitssphäre (z. B.: das Ethische, der Lebenstil, die Geschlechtsliebe), in die Sozialsphäre (z. B.: das Politische). Der dritte Teil endlich, der konkreteste würde auf Grund der in den beiden ersten gewonnenen Begriffe die einzelnen Persönlichkeiten, Völker, Zeitalter, Zustände analysieren. Hier hätte Systematik keinen Sinn, Monographien müBten an ihre Stelle treten (40),

Bei der Einteilung der Einstellungen wie der Weltbilder wird in einer an Schelling'sche Schemata gemahnenden Art von neuem der Gegensatz von Subjekt and Objekt verwendet. Diesen Gegensatz vereinigt dann eine dritte Form in eigentümlicher Weise. So entstehen gegenständliche, șelbstreflektierte and renthusiastische Einstellungen, sinnlich-räumliche, seelisch-kaltarelle und metaphyșische Weltbilder. Die dritte 'Form sucht dabei stets die zwei ersten zu vereinigen, kann däs aber nicht in einem festem Gebilde, sondern nur in lebendig̀er Bewegung leisten; diese Dynamik führt notwendig za den:Geistestypen äls Ganzen, in denen sich die lebendigen Kräfte oder "Ideen" offenbaren.

Der "begrenzte Raum dieser Zeitschrift zwingt mich, die Anảlȳse auf dịe Geistestypen zu beschränken. Zu ihrem Verständnis aber ist es nötig, sich über die Wertungen begrifflich žù orientieren. „In den Wertungen, dịe die Kräfte des Lebens sind, ist etwas Letztes gegeben. Warum jemand werten solle, ist auf keine Weise etwa objektiv zu begründen. Der Mensch tut es, sofern er lebt; er kann sich seine Wertungen klären, sie formulieren, objektivieren, aber "erst müssen sie da sein und erfahren werden" (190). Am Werte unterscheidet J. (191) den "Wertträger " und den "Wẹrtakzent". Der Wertakzent hat g’egensätzlichen Charakter, tritt als Forderung an das Subjekt heran und gewinnt mannigfaltige Gestalten je nach dem Organ, an das er sich wendet, (Gefühl, Urteil, Wille) sowie nach seinem eigenen qualitativen Charakter (Lüst, gesund, schön, richtig u. s. f.). Infolge dieser Verschiedenheit der Wertakzente and infolge der realen Eigenschaften der Wertträger entstehen Kollisionen der Werte, deren 
Entscheidungen sich in Rangordnungen der Werte objektivieren (192). In den Wert-Kollisionen, aber nieht nur in ihnen, erscheint dạs : Leben ebenso als Wertvernichtung wie als Wertschöpfang. Diese Wertvernichtung wird in unzähligen Situationen erfahren, die als einzelne zufällig sein mögen, doch aber ihrer Gattung nach mit dem Menschsein notwendig, verknüpft sind. Sie treten an den Grenzen unseres Daseins auf, darum nennt J. sie Grenzsituationen (202). Ihnen gegenüber verzweifelt : der Mensch, falls er nicht - wie durchaus die Regel ist - irgendwo seinen Halt hat, Welches dieser Halt ist, das ist der charakteristische Ansdradk der in dem Menschen lebendigen Kräfte. Daß die Grenzsituationen, obzwar im einzelnen Fall vielleicht überwindlich, doch notwendig: wiederkehren, darin zeigt sich die "antinomische Straktar" der Welt (203). „Reale Gegensätże sind Antinomien, wenn sie als etwas Lețtes aufgefaßt werden, das vom Standpunkt des Wertens' aus als wesentlich and fragwürdig erscheint, und wenn die Existenz als im Letzten in Gegensätze entzwiteit gefaßit wird; so daß alles einzelne Dasein nar dann besteht, wenn diese gegensätzlichen Kräfte oder Erscheinungen sich zusammeñfinden " (205). Diese Antinomien sind „völlig evidente Realitäten, in denen wir leibhaftig existieren ". Sie setzen das Erkennen, dessen Grenze sie bilden, zugleich in Bewegung. Dagegen ist, es der Tod des Erkennens, wenn man fertig formulierte Antinomien als Erkenntnis ansieht. Das Erkennen lebt nur in konkretem Fortschreiten und erfährt die Antinomien nur in diesem Fortschreiten (209). Die Antinomien können den Menschen zerstören, sie können von ihm umgangen werden ( in ihnen Kraft gewinnen, sein Einheitswille kann eine Synthese erreichen. Aber: „Die Synthese der Antinomien existiert nur als lebendiger Akt, unendlich and rätselhaft für den Lebendigen und unendlich anch für die Analyse, die der Betrachtende daran versucht. Die Synthese ist bloBe Spielerei, wenn sie intellektaell in Formeln geschieht" (213).

Alle Grenzsitnationen werden als Leiden erlebt. Der Reflexion bleibt die antinomische Struktur, somit das Leiden, das Letzte, während das Positive, die Last, dem aktiven Leben zugehört, nar darch Appell an das Leben erreichbar ist (219). Anf das Leiden reagiert der Mensch im einzelnen entweder so, dab er es als vermeidbar, oder so, dab er es als endgültig anffabt. Das erste geschieht, wenn er dem Leiden ausweicht oder sich durch 
endliches, technisches Tun aus dem Leiden hinausreißt oder es bekämpft in der (im einzelnen Falle oft berechtigten, im Ganzen unmöglichen) Hoffnung es besiegen zu können, oder endlich indem er es in ein Gates umformt, sei es im Reśsentiment, dem die eigene Unwertigkeit als das Höhere erscheint, sei es in der Rechtfertigang eigenen Glückes, wo das Leid·anderer zur Strafe wird (221 f.) Diesen Formen des Optimismus steht nicht nur der Pessimismus sondern auch eine beide ablehnende Lebendigkeit gegenüber (219f.).

Die Grenzsituationen zeigen die Lage des Menschen als antinomisch, und dieser Tatsache entspricht der sich immer wiederholende ProzeB seines Lebens. Er sucht gegenüber dem Zerstörenden einen Halt, der zu einem festen „Gehäuse" wird. In ihm lebt er selbstverständlich, bis die bewußte Erfahrung der Grenzsituationen eine grenzenlose Dialektik der Reflexion erzengt. Dadurch wird das Gehäuse aufgelöst, aber: „daß der Mensch lebt und nicht zu Grunde geht, ist daran sịchtbar, daß er im Auflösungsprozeß des alten Gehänses gleichzeitig neu Ge Gäuse oder Ansätze dazu baut". .... „Der Prozeß dieses Nachanßensetzens, ist das Leben selbst" (249). "Wird dies 'gewuBt, so kann der letzte Halt im unendlichen Prozesse des Lebens sëlbst gesucht werden. So. entstehen drei Grundstrêbungen: ein Drang $n$ in unendlicher Verantwortung, Iebendigem Wachsen und Schaffen zu erfahren, was das Dảsein , sei, und es darin zugleich selbst mit za gestaltẹn", ein Drang ins Nichts und ein Drang ins Gehäuse. Jede dieser Strebungen ergibt, wenn sie herrscht, eine Reihe von Geistestypen, die J. schildert unter den Titeln: Skeptizismus and Nihilismus, der Halt im Begrenzten, der Halt im Unendlichen. Es fällt zanächst auf' daß 'die negativiven Richtungen vorangestellt werden, während sie doch von der Auflösung der Gèhäuse leben - aber die Reihenfolge rechtfertigt sîch ans, der Einschränknng auf individuelle Weltanschaungen, in der J. sich seine Aufgabe stellt. Denn solche entstehen erst, "wenn der Auflösungsprozeß sich in einer Gèsellsčhaft verạllgemeinert.

Vollständiger $\mathrm{Ni}$,hilismus kann im Leben nicht existieren; so lange der Mensch lèbt, wird inmer noch irgend etwas als positiv festgehalten, etwa das nackte Dasein einer sinnlosen Realität rom praktischen Materialisten, der alle Wente negiert (Wertnihilismus), der Wert und Sinn vom Buddhisten, der die Realität als wertwidrig verwirft (Seinsnihilismus) (252f.). Die konkreten Gestalten unterscheiden sich danach, ob der Mensch sich gegen den Nihilisinus 
wehrt, dessen er nicht Herr werden kann, oder ob er mit dem Nihilismus eins geworden, in ihm als in seinem Element existiert (257).

Der Halt im Gehä use kann unmittelbar, naiv sein oder als Selbsterhaltung gegen den drohenden 'Nihilismus - willkzürlich gewählt. Auch im zweiten Falle kann der Mensch wirklich im Gehäuse leben, ohne darüber zu reflektieren, aber êr behält. im Instinkt Angst vor der Reflexion. Die gewachsenen Gehätuse des 'Naiven bilden sich um, sie sind lebendig, die gewähiten des Flüchitlings sind fertig, mechanisch, tot (27.0). Diese zweite Form, die hier ja allein betrachtet wird, ist bei aller inhaltlichen Verschiedenheit der. Gehäase, zusammengehalten durch den Rationalismus. „Der Rationalismus ist der Geistestypas, der im Begrenzten und Begrenzbaren, im Fixierbaren und Endlichen verharrt, der mit dem Verstande alles faßt und darüber nichts mehr sieht" (271). Er überwindet sich selbst, nicht durch bequemen Verzicht aúf das Begreifen, sondern indem er sich bis zọm Änßersten exweitert und dabei seine -Eigenschaften und Grenzen: erkennt "(272)., In das. echte Leben des Geistes gehen Auflösungsprozesse and Gehäase ein, es ist aber selbst unbeschreiblich; denn alles Leben isst unéndlich. Das gilt vom Leben des Leibes gegenüber jeder, auch der komplixiertesten Maschine und ebenso vom Geîste im Gegensatze zur bloßen Endlosigkeit chaotischen Seelenlebens und zu der Endlosigkeit oder Begrenztheit seiner einzelnen Produkte und Erscheinungen (289). Von solchem echten geistigen Leben könnnen wir nur reden, wo eine Richtung der Bewegung in die Unendlichkeit führt. Auch die Freiheit des Geistes ist immer nur werdend, wachsend, nie vollendet da; sie erscheint, wo ein Sinn nicht als änßere Pflicht anfgenommen wird, sondern àus dem konkreten Gehalt der Seele and der bestimmten Lage anfleuchtet. „Frei sein, heipt, ans der Totalität existieren; die Totalität aber soll erst werden " (292). Der Prozeb des Geistes hat irrationale Wendepunkte, an denen, wenn auch nach langer Vorbereitung, Erwägang ein entscheidender Entschluß ans Instinkt oder Eingebung herans gefaßt wird (294f).). Im Gegensatze za der im Letzten immer relativen Erkenntnis tritt dabei der unbeweisbare Glauben an den letzten Sinn in irgend einer Form auf. Auch der Glaube in dieser tieferen Bedeatung des Wortes' gehört erst dem Geiste an, der gegenüber den Gefahren des Negativismus und der Verengang in erstarrten Gehänsen seinen Halt im Unendlichen sucht. "Mit dem Glanben ist dialeltischer Fluß, unendliche Pro- 
blematik, Verzweiflang und Angst verbunden, weil allem Leben des Geistes die nihilistischen Bewegangen ein Element und immer eine Möglichkeit sind. Die Ungeistigkeit kann sich objektiv sicher in absoluten Gehäusen fühlen. Der Geist kann in der Angst der Bewegung nur kraft des Glaubens existieren" (298). Der Geist smacht fortwährend die Bewegang zur Klarheit und Durchsichtigkeit in der Subjekt-Objektspaltung - dieser Klarheitsdrang ist seine Feindschaft gegen alles Dunkle, Schwärmerische, das im Qualm der Undurchsichtigkeit sich wohl fühlt - aber er hat doch zum Ausgangspunkt, wie zam Ende das Mystische" (305). Der Geist wird nun zuerst betrachtet als auf dem schmalen Grate zwischen Gegensätzen wandelnd. Auf diesem Grate erscheinen als Synthesen die Gestalten des Geištes, die Ideen sind, der Realist, Romantiker, Heilige (381 ff.) - von einander verschieden durch die Art der Realität, in der sie leben. Unter den Gegensätzen sind die wichtigsten der zwischen Chaos und Form und der zwischen Vereinzelung und Allgemeinheit. Z Zwischen Chaos und verfestigter, erstarrter Form gibt es ein Drittes. Man erkennt dies im Denken, wenn man das Problem der Kónsequenz stellt. Der Denker soll konsequent sein, daś Kompromiß ist eine Schwäche. "Gehäuse schafft nur der konsequënte Denker. Aber wenn die Gehänse sich lösen, dann' entstehen irrationalistische, fragende, erregende Philosophen. Das Resultạt eines solehen Umschmelzungsprozesses ist eine antinomische'Synthese. „Es ist keine formallogische Synthèse, die ein Kompromiß ist, sondern eine psychologische, die einen neuen Ausdruck für Prinzipièen findet," ... (312). Frühere Widersprüche werdẹn nun nicht mehr erlebt, dafür aber neue; denn die Antinomien bleiben bestehen, Es gibt also dreierlei: das Verfahren logischer Konsequenz, das chaotische Neben- und Nacheinander dér Widersprüche und die Ũmschmelzung in den Prinzipien. Diesen drei Verhaltungsweisen entsprechen 3 Menschentypen: der chaotische, der konsequente und der dämonische Mensch. Der Begriff des "Dämonischen " ist dabei so erweitert, dah auch Jesus unter îhn fällt. Iọ habe den Eindrūck, daß bei der sehr fesselnden Schilderung des Dämonischen zwei Typen nicht genug geschieden werden: der dämonisch Getrìebene, dem es nirgends Rüh läßt, . der sich im Fortbilden anfzehrt, den seine Kraft beherrscht (Kleist, Nietzsche) und der in tiefer Klarheit Schaffende, der seinen Dämon beherrscht and im Weiterschreiten Qual and Glück findet, (Lionardo, Goethe, Kant - nnd wenn man ihn hier nennen 
-darf: Jesus). Im ersten ist das Errangene nar Richtung, Aphorismas, Ausdruck, einzeln-fragmentarisches Gebilde, im zweiten wird es zur lebendigen Gestalt. Natürlich gibt es Übergänge, já vielleicht gehörti jedes Genie des zweiten Typs teilweise anch dem. ersten $a n_{\text {, }}$ sei es in den Anfängen (Goethe), sei es gegen Ende ! seines Laufs (Miehelangelo), wie anch diè Größten der ersten Art mit einzelnen Gebilden in die zweite hinüberreichen. Wohl kann man mit Jaspers aüch Kants Werke "riesenhafte Fragmente " (317) nennen, aber sie sind es doch in anderem Sinne und Stile als die Nietzsches. Das dämonische Leben soll nün in großen Philoșophịen als Lehre erfaßt werden. Hegels System, das hier das entscheídende Beispiel bietet, scheitert, wo es mehr als Betrachtung sein will. Denn um das Leben za fassen, muß es das Leben abgeschlossen denken - wird Kontemplation, verantwortangsloser Quietismus (328).

Um den Konflikt zwischen dem Individuum (dem Selbst) und dem Allgemeinen za verstehen and die Geistestypen, die in Kampf and Synthese sich ansdrücken, zu kennzeichnen, wैerden die verschiedenen Arten des Allgemeinen 'sorgsam untersehieden. Ich kann hier auf diese gehaltreichen Aựfühuhrungen nur hinweisen̆. Das Problem des Selbst" hat Kierkegaard am tiefsten erfabt; $\because$, seine Sätze stellt J. systematisch zusammen (370ff.).

Alles Leben des Geistes enthält als Leben Irrationales, dém von der Subjekt-Objekt-Spaltung noch ungeteilten Erlebnisstrome Angehöriges. Dies Ungespaltene, das auch ganz banale Erlebnisse umfaBt, deckt sich mit dem Mystischen im weitesten Sinne dieses Wortes (388). In unserem Seelenleben gibt es einen Prozeb von unklarer Gemütserfülllang zu klarer Vergegenständlichnng (389), alles vollkommen Vergegenständlichte ist uas bequemer Besitz, damit aber auch tot and langweilig (390). Zu mystischen im engeren, höheren Sinne werden Erlebnisse ohne Subjekt-Objekt-Spaltung erst dadurch, daB sie der Lebensgrand sind, auf das gesamte Seelenleben wirken (393). Diese in engeren Sinne mystischen Erlebnisse kömnen nun eine dreifache Rolle spielen: 1. Drang zum Mystischen, weil es als solches spezifische Befriedigung gibt, Mystik im engeren Sinne; Gefahr sich im Ausruhen abzustumpfen, in asketische Techniken za verlieren, kaltarlos za werden. 2. Drang vom Mystischen fort, das als Schwärmerei abgelehnt wird. Endloses Denken, Handeln, Schaffen in der gegenständlichen Welt. Positivismns. Gefahr, das Mystische ganz za verlieren. 3. Synthese beider Tendenzen: aus dem Mystischen geht der Drang zum Gegenständlichen and 
kehrt immer za neuem Mystischen zarück“ ${ }^{\alpha}$.. „Durch die Unendlichkeit des gegenständlichen Tuns, Denkens, Schaffens wird in fortschreitender Spirale der Kreis immer weiter, das Mystische immer neu, immer tiefer, als Ausgang weiterer Gegenständlichkeit (Entfaltung der Idee) ${ }^{\prime \prime}$ (394). Die Ausbildung des ersten Typs zarWeltanschanung läBt sich an P lotin, die des dritten an $\mathrm{K}$ ant darstellen.

Überblickt man den Umkreis der in dem Bache geschilderten Gestalten, so fällt bei allem Reichtum, die große Einengang gegenüber Hegels Phänomenologie des Geistes anf. Es fehlen ja alle nnaiven" Weltanschaungen, alle, die im "wachsenden Gehäuse" einfach leben. Eng damit ,zusammen hängt eine zweite Einschränkang der Aufgabe: J. verzichtet darauf, die Weltanschanungen als wirkende Mächte in den Seelen derer zu verfolgen, die sie nicht original hervorbringen. Man kann, seiner Ausdrucksweise nahe bleibend sagen: die Weltanschauungen werden von ihm nur soweit betrachtet, wie sie Ausdruck der Kräafte and Ideen sind, nicht soweit sie selbst als kraftbegabte Wesen fortwirken und sich in ihrer Wirksamkeit wandeln. Nun ist solche Beschränkung zanächst einfach festzustellen; denn jeder Autor hat das Recht sein Thema abzugrénzèn. Doch fragt sich, ob die Abgrenzang nicht trennt, was sich nur vereint verstehen läßț. Jeder der großen Schöpfer einer Weltanschaung steht unter dem Einflub älterer Gestalten; ohne den Prozeß der Aufnahme und Umbildung des Überkommenen zu verfolgen, kann man das Werden der Weltanschaunngen nicht vollständig begreifen. Wenn dabei der Schöpferische überzengt ist, in einer überlieferten Weltanschanung zu leben (und das ist z. B. jeder, der sich als Christ fühlt), dann bleibt auch der höchst Differenzierte (Kierkegaard z. B. oder.Pascal) noch ,irgendwie "naiv". Es ist daher unmöglich, den schöpferischen Menschen ganż žä verstehen, wenn man nicht das naive Wachstum and, was daron untrennbar ist, das Weiterwirken der Weltanschauungen verfolgt. $J$. ist zu geneigt, Originalität und Echtheit gleich zu setzen, an anderen Stellen $(343 ; 359)$ Ëchtheit mit Weite und Klarheit des Blickes zu verwechseln. Aber és gibt „echte" Jünger, denen "Treue die Person wahrt", ebenso wie es echte Dürftigkeit gibt, die gar nicht Fülle sein will, and Undifferenziertheit, die sich im Unklaren, Verworrenen echt auslebt. Wenn es anch eine Psychologie der Weltanschanungen nur auf dem Standpunkt des modernen, hochreflektierten BewaBtseins geben kann, so bedentet das doch nicht, daß dieses Bewaßtsein der einzige Gegen- 
stand einer solchen Psychologie ișt. Von hier ans kaṇn man füglich auch in der überwiegenden Benutzang Nietzsches and bes:" Kierkegaards eine gewisse Einseitigkeit sehen; denn 'so" groß Kierkegaard als Psychologe ist, seeili Gegenstand ist ausschließ̉lich der moderne Mensch. Im übrigen ist es keines der geringsten Verdienste des Werkes, daB Kierkegaard's psychologische. Einsichten aus dem religiösen und künstlerischen. Zụsammenhange gelöst und̉ in ihrer wissenschaftlichen Bedeutang èrscblossen werden.

Über die verstehend-beschréibende Psychologie der Weltanschaunngen geht $J$. in seiner Ideenlehre za einer verstehenden Erklärung fort. Er deutet dafür Kants Gedânken in eigenartiger Weise und bildet sie weiter (im „Anhang“). Die Idee hat ihr Wesen” in der Totalität and Unbedingtheit. Von dem Ganzen her gewinnt J. eine neue Ordnang der Ideen (413); denn "dieses Ganze kann entweder ein Ganzes von Erfahrungsrichtangen oder ein Ganzes von Erfahrungsinhalten sein. So entstehen zwei Klassen von Ideen: Dex ersten, den Ganzheiten von Erfahrangsrichtangén gẹiören za: Mechanismus, Organismus and Seele. Mit der dritten dieser Ideen hat sich Kant nicht genauer beschäftigt. $J$. sieht in der Idee der Seele eine ganze Reihe von Ideen, neben Mechanismus und Organismas, die auch hier auftreten, zwei neue Formen: „die'Idee des Ganzen der exlebten und erlebbaren Phänomene oder die Idee des Bewabtseins and vor allem die Idee des Ganzen der verständlichen Zusammenhängé, oder die Idee der Pers önlichkeit" (417). Unter den Ideen vom Ganzen des Erfahrungsinhaltes behandelt J. nur die des Einzeldings als einer Unendlichkeit näher. Diese Idee läßt sich auch anf die Persönlichlreit anwenden, so daß neben der Idee der Persönlichkeit als Erfahrungsrichtung eine zweite Idee der einzelnen konkreten Persönlichkeit erscheint (419).

Man kann Ideén nur dadurch erfassen, daß man in ihnen lebt, theoretische also nur im Leben des Verstandes. „Der Verstand steht zwischen zwei Irrationalitäten, ohne. die er leer ist, die aber ohne ihn nichts sind. Er ist hingewandt anf die Breite der Anschaulichkeit des Materialen and bewegt von den Kräften der Ideen. Das Anschauliche geht als Irrationales über den Verstand hinaus, aber wird von seinen Begriffen umfaßt. Die Ideen gehen über den Verstand hinans, indem sie seine Grenze, ihn selber umfassen; seine Begriffe können die Ideen nicht einfangen, sondern nur aaf sie hinzeigen" (420). Abweichend vom Kantischen Sprachgebranch faBt J. alles, was nicht Verstand ist, als Anschanung zusammen 
und unterscheidet dann eine materiale, stoffgebende Anschaung von einer ideenhaften, Kraft and Bewegung gebenden, die nur erlebt, nicht erfaßt werden kann. Die Ideen haben drei, von Kant nicht scharf getrennte Bedentangen, eine methodologische, eine psychologische, eine metaphysische. Von der ersten als der bekanntesten, brancht kaam weiter geredet zu werden. Als psychische Kräfte geben die Ideen der Wissenschaft Richtung und Tiefe. Sie sind, wie Kant ausführt, oft wirksam, ohne erkannt zu sein. J. sagt (422): „Es ist merkwürdig, daß wir in der Wissenschaft volle Darchsichtigkeit und Klarheit wollen and daß doch, wenn diese bis zum Letzten vorhanden ist, unser Interesse erlahmt. Wir wollen K̇larheit, aber wir wollen, daß sie der teilweise Ausdrack einer Idee sei. Diese Idee ist in der wissenschaftlichen Leistung als das Donkel vorhanden, das ebenso sehr verständnislọsen Angriffen arasgesetżt wie Bedingung ihrer produktiven Wirkúng ist". Die objektive, mètaphysische Bedeutung der Ideen wird durch den inegativen Aufbau der transzendentalen Dialektik verhüllt, in Nebensàtz und Anhang verwieseñ. Aber man könnté sich ebensowohl einen positivien Aufbau der Dialektik denken, der das Negativè in den Anhang, bạnte (425). Die logische richtanggebende (regulative) Bedeutung der Idee ist ihre Auswirkung; nicht ihr Wesen. " Zu den Ideen verhalten sich die Weltanschauangen als Äußerungen, sie sind daher, sobald man sie formuliert, nichts Letztes, "bleiben relativ. Die Ideen "als das Letzte, könntien alśs. daș Absolutte beźeîchnet werden (wenn aúch nur für den Kreis der Bettrachtung), sie sind das Leben selbst, das nie ganz und gär äußerlich; objektiy wird, wenn auch immer dahin drängt" (25):

Die Beziehuing von "Idee " und "Lieben", die hier angedeatet ist, "hat $J$ nirgends genau bestimmt. Geht man in Richtnng seiner "Gedanken weiter, so entdeckt man ein verwickeltes Netzwerk von Beziehungen, die sịch in żwei Gruppen ordnen: Leben selbst ist für das theoretische BewaBtsein "Idee ${ }^{2}$ und Idee ist Leben des Geistes, d. h. zum Selbstbewabtsein strebendes" Leben. Nimmt man den letzten Ausdruck ernst, dann sieht man in allem Leben (mit Sehelling) die "Idee" wirksam - die Natur wird als lebendige zur ,Odyssee des Geistes"." Von Schelling unterscheidet sich J. durch seinen bewnßteren Irrationalismus : es wird anerikannt, daß eine streng begriffliche Darstellung oder gar ein logischer Beweis hier nicht möglich ist. Aber von anderen Irrationalisten hebt sich 
J. dadurch ab, daß er die 'Notwendigkeit der Ratio, die Pflicht,' so weit möglich Klarheit ins Dunkel za bringen, erkennt. Bei einer ganzen Reihe von einander unabhängiger Denker finden sich heute Ansätze zu einer ihrer selbst nicht ganz sicherẹin Metaphysik des Lebens. Sie tritt bei Spengler mit der Geste der Genialität auf, bei J., der durch Lask's und Husserl's Schulè gegangen ist, mit kritischer Scheu. G. Simme1, der einer Erkenntnis des Lebens bisher vielleicht am nächssten, gekommen ist, ' hat ' in seinem

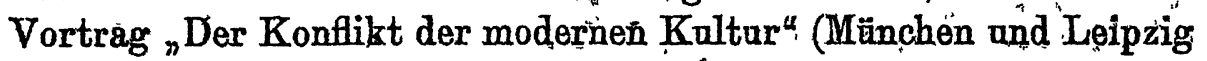
1918) auch die geistesgeschichtliche Errkenntnis dieser Strömung am meisten gefördert. Man wundert sieh, daB Simmel in 'J.'s Buche nicht genannt wird.

Da J.'s Háltung bei aller Hinneigung zưr Metaphysik kritisch bleibt, sucht er sich über die Art und Tragweite der angewandten Erkenntnismittel Rechenschaft za geben und sich innerhalb der Grenzen zu halten, die sie ihm anszafüllen erlauben, nur daß die. Grenzen des Erkennens ihm nicht Grenzen des Lebẹns bedepten and dab das Erkennen selbst, da von Ideen geleitet, einen urerkannten Lebensgrund in sich trägt. Mittel. des Erkennens von Weltanschaungen ist wesentlich der Prozeß, den man "verstehen" nennt, und am dessen Aufhellung seit Dilthey die Denker bemüht sind, trotz aller feinen Bemerkangen noch ohne vollen Erfolg. Verstehen ist nur möglich auf Gqund eigener Erfahrung. Unsere weltanschanliche Erfahrung aber ist ein BewegungsprozeB. Nur weil wir und solange wir in dieser Bewegung leben, können wir Psychologie der Weltanschanungen treiben. Ist uns alles fest. geworden, dann besteht kein Interesse mehr dafür, es sei denn als für eine Psychologie der Täuschungen (7). Da verstehende Err-' kenntnis aus eigenem Erleben stammt, ist sie nur nacherlebbar, nicht beweisbar (13). Die Grenzen der Geltang dieses Satzes hätte $J$. finden müssen, wenn er untersucht hätte, wie sich die Einsicht in sachliche Zusammenhänge zum Verstehen verhält. Fr hätte Anlab dazu gehabt, da er von dem, was Max Weber einmal "rationales Verstehen " genannt hat, vielfach Gebranch macht, z. B. überall da, wo er die Antinomik der menschlichen Lage, das Ungenügende jedes festen Gehäuses heranzieht.

Hänfiger als anf die Theorie des Verstehens geht J. anf die Dialektik als anf ein Mittel der Darstellnng des Lebens ein. Die Dreiteilnngen, die das Werk beherrschen, sollen nicht etwa Entwicklangsreihen sein, soda $B$ es mit der Thesis anfinge, mit der 
Synthesis ende. „Vielmehr ist es ein Herumgehen um ein Ganzes, das erst in Gegensätzen, dann selbst ins Ange gefaßt wird. Man könnte ebensogut sagen, das Ganze stehe am Anfang and der Gegensatz der beiden ersten Teile entfalte sich daraus ${ }^{4}$... „Das Greifbare liegt immer im ersten und zweiten Teil, hier liegen die faktischen, sichtbaren Mannigfaltigkeiten, das Dritte ist das Dunkle ${ }^{\text {" }}$ (26/7). Die dialektische Folge ist eine bloße Ordnung der Begriffe, kein System des Lebens und Daseins selbst. Die Dialektik gibt dem Denken vor allem die "Bildung“ (69), d. h. sie ist unfähig Erkenntnïsse za schaffen, weiß aber' die Zusammenhänge der Begriffe darzustellen and damit die Erkenntnisse für die Person fruchtbar zu machen. Aus dieser Umgrenzung folgt J.'s Stellungnahme zu Hege1. Er gibt zu, Wesentliches von ihm gelernt zu haben; aber dạ die Synthese dúnkèl bleibț; so behält die einfach antinomische Struktur, wie Kant-sie dargelegt hat, neben der synthetischen Form ihr Eigenrecht., Damit hängt dann eng zusammen die Ablehnung von Hegels These; daß Denken und Sein eines sei; dialektisches, Denken umkreist nar die Anschaülichkeiten: Indem Hegel die Idee in der Dialektik objektiviert; tötet er sie (326). W' Hegel "bloße' Betrachtang gibt, ist er 'ungemein fruchtbar, wo er àls "prophetischer" Philosoph Weltanschaung lehren will, versagt.er. : Seine Lehre ist nicht schöpferisch, sondern findet sich rechtfertigeñd mit dem $a b$, was, durch andere Wirklichkeit geworden ist; wird ' $n$ kiontemplativê, , veräntwortangsloser Quietismus". Daram wúrde diese Philiosophie vọm Leben ausgestoßen.

Zw̦ei Sätzè, 'dile' sich âs J.'s Stellungnahme zar Dialektik entwickeln lassen, sind richtig und fruchtbar: 1. das dialektische Denken allein hat die Mittel, den LebensprozeB des Geistes darzuștellen.' 2. 'Dèr Fornmalismus der Dialektik ist ebenso wenig 'wie irgend ein anderer Formalismus fähig, aüs sich heraus Einsichten zu erzeúgen. Freilich; dieser z"weite Satz, als negativer, bedarf' der positiven Ergänzang: es ist die Bedeutung sowohl der immanenten Dialektik wie dér Selbsterkenntnis der Dialektik für das Erkennen zu entwickeln. Der erste Satż enthält dazu nur die Anweisung. Wenn J. sagt, daß die Dialektik um das schaffende Leben des Geisteś nur herumgeht, so ist das erstlich ein bloßes Bild, desisen Wahrheitsgehalt zu entwickèln wäre, und zweitens erschöpft es die Bedeutung der Dialektik ganz gewiß nicht.

J. selbst hat" an manchen Stellen durchaus nicht dialektisch genug gedacht, so nimmt er z. B. die Auflösung der Gehäuse viel 
za wörtlich - als blieben etwa nur die Mațerialien übrig, während in Wahrheit gerade der Bauplan sich in rimgebilleter Form erhält, solange überhaupt noch Kontinuität des Einzellebens oder der Kaltur besteht. Ja Auflösung befireit zugleich wesentlichen Gthalt: Jeder in einer Dialektik vorkommende Begriff ist dialektisch za" behandeln. Eine Theorie der Dialektik hätte unter anderem das za erweisen and darchzuführen. Sie hätte neben der "Hegel'schen auch andere Formen der Dialektik ,zu untersuchè. Auch ohne daß eine solche Theorie vorliegt, 'könnte z. B. von Schleiermacher für die Psychologie der Weltanschaungen vieles gelernt werden. Am meisten leidet darantex; daß J. die Dialéktik nar untęrgeordnet verwendet, die Selbsterkenntnis seines eigenén Tans and seiner Stellang in der Wissenschaft. J. behauptet immer wieder, daß er lediglich wertungsfreie Betrachtung erstrebt. "Er grenzt seine Aufgąbe, Weltanschaungen zu verstehen; bescheiden ạb gégen das Ausbilden einer Weltanschanung, das Sache der "prophetischen-Philosophie ${ }^{\prime \prime}$ ist, and er verwahrt, sich mit dem ganżen Stolze strenger, resignierter Wissenschaftlichkeit dagegen; mit den unechten Surrogaten einer prophetischen Phílosophie, verwechsielt zn werden, die man heute überall herumbietet. Dem gegenüber sind der Psychologie (auch der verstehenden) die Werte lediglich Gegenstand. Natürlich soll das nicht bedeuten, daß" der Psychologe als Mensch sich des Wertens enthält $\rightarrow$ so wenig etwa der Botaniker als Gärtner Unkraut und Gartenblume gleich behandelt. Aber die Fälle des Botanikers und des Psychologen liegen nicht gleich: dem Botaniker ist die Pflanze ein gesonderter Gegenstand, den er mit einer von jedem erlernbaren Methodik untersucht, der verstehende Psychologe erkennt mit seinem ganzen Leben - der. Reichtum nachfühlenden Verstehens hängt von dem Reichtum des Eigenlebens ab. J. übersieht diesen Einwand nicht, er bemerkt auch, daß gewisse Wertungen sich schwer ausschalten lassen (z. B. S. 154). $\mathrm{DaB}$ z. B. das Leben gegenüber der "Unlebendigkeit", die "echten" Gestalten gegenüber den "unechten ${ }^{\prime}$ durch einen positiven Wert hervorgehoben sind, weiß J. natürlich (vgl. bes. 280). Aber J.'s wertende Stellungnahme reicht viel weiter. Ganz deutlich strebt er einer Weltanschaung zu, die in der vollen, gestaltenden Lebendigkeit das Wesentliche sieht. „Es gibt zwischem dem Nihilismus und dem Gehänse, zwischen dem Chaos and der Form ein. Leben ans dem Ganzen and Unendlichen, das nicht kompromißlerisch, halb and wesenlos ist" (308). Er weiß, daß die bloBe Bejahnng 
des Lebens, des Dämonischen diese Weltanschaunng noch nicht gibt (329) - aber welchen Sinn hat diese Bejahung als den des Willens za einer solchen Weltanschaunng? Ja an mehreren Stellen zeigt $J$. das volle Bewußtsein der Bedeutung, die die Psychologie der Weltanschaungen für die Erringung einer Weltanschaung aus wahrhafter Anschaung des Lebendigen besitzt. So heißt es einmal von der psychologischen Betrachtung, sie sieht in der Kraft des Gehäusebans Kraft des Lebens und damit das Wesentliche. „Sie weiß, daß sie selbst leicht ein Faktor im AuflösungsprozeB ist, $d$. h. aber ein notwendiger Faktor im Leben, damit es immer wieder zur Entfaltung komme, und d.h. eine Kraft der Auflösung, der schließlich gerade nur die unechten Gehäusefabrikate oder die überlebten Versteinerungen und die kraftlosen, lebenslosen Menschen anheimfallen, wie die Bakterien sich aller Leichen aber nicht der lebendigen Leiber bemächtigen. Sie darf erkennen, daß sie zwar ihrem Wesen nach selbșt unschöpferisch ist, daß sie aber im Dienst des wachsenden Lebens steht, dem, ein Schaden durch sie zuzufügen "unmöglich ist" (249f.). Aber J. will nicht sehen, dạb seine Wertung des Lebens keineswegs nưr gelegentlich mitschwingt, sondern die 'Gestalt seines Buches bestimmt.' Ein. Mensch, der überzengt ist, dab sei es eine bestimmte gegebene Form (z. B. die des Katholizismus), sei es das. Leben in fester Form überhaupt das Rechte und Wahre ist, würde nie von "Gehäusen" reden, die aufgelöst and umgebildet werden. Wer das Leben negiert, wie Bưddha oder Schopenhaner, wird alle Erkenntnis des Lebens nur als Mittel ansehen, sich vom Leben abzukehren: Es ist anffallend, wie gering der Raum ist, den J. den negierenden Geisteshaltungen widmet." Die strenge Abkehr Büddhas wird nur gestreift, die realistische Verzweiflung Bahnsens gar nicht erwähnt. 'Auch die Ausschaltung der naiven Formen gewinnt so eine andere Bêdentang 'als die ihr Jaspers geben will: sie stèhen noch diesseits des 'entscheidenden Prozesses. Es könnte scheinen, dies seien nur Mängel 'der Dúrchführung seines Planes. Aber daran wird man irre, wenn man J. mit Hegel vergleieht. Hegel "hat in seiner Phänomenologie ausgesprochen das Ziel, die höchste Stufe des Bewußtseins zu erreichen àuf dem "Wege des Durchgangs durch die ganze Entwicklung des Geistes:. Dabei kommt jedes Entwicklungstadium in seiner Eigenart zu Recht; es ist die Aufgabe des Philosophen, sich ganz mit dieser Gestalt zu vereinigen, um sie aús sich selbst heraus za überwinden. J. will jede Gestạlt nur betrachten 
- und dabei drängt or viele Gestalten ganz in den Hintergíand zugunsten weniger, die sich als Näherungen an ein ihm selbstrerständliches Ziel auffassen lassen. Es zeigt sich: die unterdrüuckte uneingestandene Wertung ist viel schädlicher fürr die Reinheit der Betrachtung als die eingestandene. Also, könnte man folgern, handelt es sich darnm, die Wertungen, die uneingestanden bei... zugrunde liegen, ans licht za ziehen, um siè unsehädlich za machen; dann aber die wertungsfreie Betrachtang so rein wie möglich durchzuführen. Man mab so folgern, weǹn man. J.'s Auffassúng des Wertes beibehält: daß der Wert nur ein Akzent ist, der anf die Sache gesetzt wird (119), daB also die Sache ohne den Wert die gleiche bleibt. Gewiß gibt es Fälle, anf die diese Beschreibung in großer Annäherung zutrifft. Ich lege keinen 'Wert auf die Schönheit der Menschen, obwohl ich diese Schönheit als solche sehe - ich lerne es, anf den Besitz von Geld Wert zu legen, obwohl mir Geld nach wie vor Mittel z. B، der Unabhängigkeit oder der Macht bleibt, kein Eigenwert wird. Aber màn erkennt an solchen Beispielen, daß hinter der Schicht, in der "Wertakzente ${ }^{*}$ äußerlich aufgesetzt werden, eine andere liegt, in der die einem. Verhalten, einer Sache, einer Person immanenten Werte gesehen werden. "Der Begriff "Leben", mindestens in dem Sinne, in dem J. ihṇ braucht, ist in sich selbst werthaltig. Wer den Wert des Lebens negiert, der weigert nicht einem gleichgesehenèn Leben einen Wertakzent, sondern er hat gar nicht den Begriff "Leben", den J. voraussetzt. Wir können nur aus einem Wertganzen heraus verstehen; wir verstehen um so besser je vollständiger in sich, je geklärter dies Wertganze ist. Die Ausschaltung der Wertungen behält ihr Recht überall da, wo eine vereinzelte Untersuchung über Tațbestände geführt werden soll. Der Eintaß einer Geistesrichtang, die Macht eines Volkes, der Erfolg einer wirtschaftlichen Bewegung sóll fest- gestellt werden ganz unabhängig davon, wie wir uns wertend dazu stellen. Solchen Aafgaben gegenüber ist als unschädlich zu vernachlässigen, was an Werten schon in der Abgrenzung der Tatbestände steckt - denn diese wird nicht untersucht und kann praktisch als zugestanden betrachtet werden. In der verstehenden Psychologie kann zwar nicht jeder Wert ausgeschaltet werden, wohl aber in begrenzender Betrachtnng jeder über die gerade $z \mathbf{a}$ verstehende Gestalt hinausweisende Wert.' Aber das ist dann bewnBte Vereinzelnng - und es ließe sich wohl zeigen, daß diese immanente Betrachtung, indem sie das ganze innere und äußere 
Verhalten der Stufe nachlebt and seine Konsequenzen zieht, auf die Grenzen, die übersehenen, negierten Werte führen müßte. Die Trennung der Werte von den Tatbeständen, so notwendig sie für bestimmte Aufgaben der Praxis und dér sich spezialisierenden Erkenntnis ist, maß doch, wie jede Trennung, vorläufig bleiben and durch eine vollständigere Besinnung auf die Verbundenheit der Getrennten überwanden werden.

An einzelnen Orten seines Werkes nähert sich J. der echt philosophischen Haltang, so überall wo er an Stelle eines abstrakten Begriffes des Psychischen einen lebensvollen setzen will (z. B. 307 Anm.). Meist aber ist eine bestimmte philosophische Anschaung nur als unbewußt treibende Kraft, als latente Idee, wenn man das Wort in seinem Sinne gebraucht, wirksam. Durch den recht schiefen Ausdruck sprophetische Philosophie" - denn

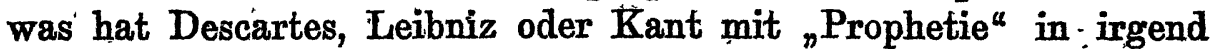
einem. Sinne zu tan? - verhüüllt er sich die Zasammenhänge zwișchen dem was er Betrachtung and dem was er Weltanschanung nennt: - Die verschiedenen Mängel des 'Werkes hängen , eng zusammen: weil J. vor der Pforte der Philosophie stehen bleiben will, während Philosophie in ihm lebt, vereng't sich ihm durch unbewaßte Wertung am falschen Platze das Bereich der Betrachtung. Weil er das Dogma von der Unmöglichkeit wissenschaftilicher Entscheidung über Werte festhält, statt die Dialektik dieses Verhältnisses zq wissen, setzt er vorläufige Trennangen als endgültig and kann zuglèich dẹn. Wert nur als "Wertakzent" fassen, sich so den Zugang zur echten Wertlehre verriègelnd. Das "Leben ${ }^{*}$ and die "Dialektik" bleibén unverbunden - statt, daß die Dial'ektik als Leben des Denkens und das geistige Lieben als denkendes erfaßt wird - so daß als die Wahrheit der Einheit von Sein and Denken bei Flegel sich die Idee einer Einheit beider zeigen kamn.

J. hätte sehr leicht ein viel einwandfreieres Buch schreiben können: er hätte nar unter Verzicht auf die Erörterung der methodischen Fragen der wissenschaftstheoretischen. Prinzipien und der philosophischen Grundlagen einfach seine systematisch gegliederten Schildernngen zu geben braøchen. E's ist zu rühmen, daß er das picht getan hat. , Denn erst durch die anfechtbaren Teile wächst "das Werk hinein in die große Geistesbewegung der Zeit, nimmt es, , besser gesagt, Teil an der Arbeit, die für Gegenwart und Zakanft nötig ist. 